\title{
OC96: An investigation into the effect of a low versus high glycaemic index breakfast on satiety and subsequent food intake.
}

\author{
N. Taylor and S. Drummond \\ Queen Margaret University, Musselburgh, Edinburgh EH21 6UU, UK.
}

Rising levels of obesity and the associated health risks, continues to be our biggest public health challenge of the $21^{\text {st }}$ century. Public health strategies have not yet been successful in reversing the obesity trend. Therefore, simple strategies to regulate food intake and facilitate the maintenance of a healthy weight have increasingly become an area of interest and research. Studies have shown that consumption of low-GI carbohydrates prolong satiety, reducing subsequent food intake. ${ }^{(1)}$ This has led to the proposal that low-GI carbohydrates could be used in the regulation of food intake to induce weight loss in the overweight and obese. ${ }^{(2)}$ However, the findings are not always consistent ${ }^{(3)}$ and the current evidence is conflicting.

This pilot study aimed to compare the impact of a low glycaemic index breakfast with a high glycaemic index (GI) breakfast on satiety over 3 hours and subsequent food intake over the rest of the day. 10 healthy female subjects (18-65yrs) took part in this cross over study. Subjects were randomly allocated to either the high or low GI breakfast on test day one, and the alternative breakfast on test day two. Breakfasts were matched as closely as possible in macronutrient and energy content. Each ingredient was assigned a GI using the international table of glycaemic index and glycaemic load values. ${ }^{(4)}$ Ingredients (cornflakes/porridge, white bread/rye bread, jam, orange juice/skimmed milk) were selected to result in either a high or low GI meal. Satiety was self reported, using a visual analogue scale and dietary intake was assessed using a self reported 24 hour food diary. Paired sample t-test was used to compare mean values.

\begin{tabular}{|c|c|c|c|c|c|}
\hline \multirow[b]{2}{*}{ 24hr Dietary Intake } & \multicolumn{2}{|c|}{ High GIycaemic Index } & \multicolumn{2}{|c|}{ Low Glycaemic Index } & \multirow[b]{2}{*}{$\mathrm{p}$-value } \\
\hline & Mean & $\mathrm{SD}$ & Mean & $\mathrm{SD}$ & \\
\hline MJ & $10 \cdot 45$ & 1.63 & $7 \cdot 90$ & $1 \cdot 16$ & $\mathbf{0} \cdot 003$ \\
\hline Kcal & 2496 & 389 & 1887 & 277 & \\
\hline Fat $(\mathrm{g})$ & 84 & 24 & 63 & 19 & $\mathbf{0} \cdot 007$ \\
\hline Protein (g) & 85 & 18 & 65 & 17 & 0.039 \\
\hline $\mathrm{CHO}(\mathrm{g})$ & 377 & 81 & 287 & 40 & $\mathbf{0} \cdot 025$ \\
\hline Satiety Ratings & Mean & $\mathrm{SD}$ & Mean & SD & \\
\hline $15 \mathrm{mins}$ & $72 \cdot 2$ & $15 \cdot 8$ & $87 \cdot 7$ & $12 \cdot 2$ & $\mathbf{0} \cdot 012$ \\
\hline 30 mins & $70 \cdot 0$ & $19 \cdot 1$ & $85 \cdot 6$ & $12 \cdot 6$ & 0.022 \\
\hline 45 mins & $65 \cdot 8$ & $21 \cdot 0$ & $82 \cdot 6$ & $13 \cdot 8$ & 0.024 \\
\hline 60 mins & $59 \cdot 2$ & $21 \cdot 5$ & $77 \cdot 1$ & $16 \cdot 6$ & 0.057 \\
\hline 90 mins & $43 \cdot 8$ & $25 \cdot 8$ & $70 \cdot 0$ & $13 \cdot 9$ & $\mathbf{0} \cdot 010$ \\
\hline 120 mins & $30 \cdot 1$ & $21 \cdot 8$ & $56 \cdot 8$ & $18 \cdot 5$ & $\mathbf{0} \cdot 006$ \\
\hline $180 \mathrm{mins}$ & $17 \cdot 6$ & $16 \cdot 1$ & $32 \cdot 0$ & $24 \cdot 2$ & 0.046 \\
\hline
\end{tabular}

The low GI breakfast elicited a significantly higher level of satiety over 3 hours and a significantly reduced dietary intake than the high GI breakfast over 24 hours. Although this was a short term pilot study, the results indicated that adherence to a low GI breakfast may be helpful for overweight individuals wishing to reduce their energy intake. However, longer term, larger studies are required to confirm these findings.

1. Warren JM, Henry CJ \& Simonite V (2003) Paeds 111, 414

2. Esfahani A, Wong JMW, Mirrahimi A et al. (2011) Life 63 (1), 7-13.

3. Esfahani A, Wong JMW, Mirrahimi A et al. (2009) J Am Coll Nutr 28 (4), 439-445.

4. Foster-Powell K, Holt SHA \& Brand-Miller JC (2002) Am J Clin Nutr 76, 5-56. 